\title{
BMJ Open Non-Invasive Positive airway Pressure thErapy to Reduce Postoperative Lung complications following Upper abdominal Surgery (NIPPER PLUS): protocol for a single-centre, pilot, randomised controlled trial
}

\author{
Jane Lockstone, ${ }^{1,2}$ lanthe Boden, ${ }^{1,2}$ Iain K Robertson, ${ }^{3}$ David Story, ${ }^{4}$ \\ Linda Denehy, ${ }^{2}$ Selina M Parry ${ }^{2}$
}

To cite: Lockstone J, Boden I, Robertson IK, et al. Non-Invasive Positive airway Pressure thErapy to Reduce Postoperative Lung complications following Upper abdominal Surgery (NIPPER PLUS): protocol for a singlecentre, pilot, randomised controlled trial. BMJ Open 2019;9:e023139. doi:10.1136/ bmjopen-2018-023139

- Prepublication history for this paper is available online. To view these files, please visit the journal online (http://dx.doi org/10.1136/bmjopen-2018023139).

Received 24 March 2018 Revised 18 August 2018 Accepted 23 November 2018

Check for updates

(C) Author(s) (or their employer(s)) 2019. Re-use permitted under CC BY-NC. No commercial re-use. See rights and permissions. Published by BMJ.

For numbered affiliations see end of article.

Correspondence to

Jane Lockstone;

jane.lockstone@ths.tas.gov.au

\section{ABSTRACT}

Introduction Postoperative pulmonary complications (PPCs) are a common serious complication following upper abdominal surgery leading to significant consequences including increased mortality, hospital costs and prolonged hospitalisation. The primary objective of this study is to detect whether there is a possible signal towards PPC reduction with the use of additional intermittent non-invasive ventilation (NIV) compared with continuous high-flow nasal oxygen therapy alone following high-risk elective upper abdominal surgery. Secondary objectives are to measure feasibility of: (1) trial conduct and design and (2) physiotherapy-led NIV and a high-flow nasal oxygen therapy protocol, safety of NIV and to provide preliminary costs of care information of NIV and highflow nasal oxygen therapy.

Methods and analysis This is a single-centre, parallel group, assessor blinded, pilot, randomised trial, with 130 high-risk upper abdominal surgery patients randomly assigned via concealed allocation to either (1) usual care of continuous high-flow nasal oxygen therapy for 48 hours following extubation or (2) usual care plus five additional $30 \mathrm{~min}$ physiotherapy-led NIV sessions within the first two postoperative days. Both groups receive standardised preoperative physiotherapy and postoperative early ambulation. No additional respiratory physiotherapy is provided to either group. Outcome measures will assess incidence of PPC within the first 14 postoperative days, recruitment ability, physiotherapyled NIV and high-flow nasal oxygen therapy protocol adherence, adverse events relating to NIV delivery and costs of providing a physiotherapy-led NIV and a highflow nasal oxygen therapy service following upper abdominal surgery.

Ethics and dissemination Ethics approval has been obtained from the relevant institution and results will be published to inform future multicentre trials.

Trial registration number ACTRN12617000269336; Preresults.

\section{Strengths and limitations of this study}

- This pilot study is a 130-patient parallel group randomised clinical trial of additional early intermittent postoperative non-invasive ventilation (NIV) versus continuous high-flow nasal oxygen therapy alone.

- This trial is measuring recruitment ability and feasibility of providing physiotherapy-led NIV and a highflow nasal oxygen therapy protocol.

- This trial standardises physiotherapy and postoperative ambulation.

- This is a pilot, single-centre study unlikely to be powered to determine treatment effectiveness.

- Results of this pilot study will assist the design and conduct of future definitive multicentre trials.

\section{INTRODUCTION}

Postoperative pulmonary complications (PPCs) are a common serious complication following upper abdominal surgery with a reported incidence of $13 \%-42 \% .{ }^{1-6}$ Development of a PPC is strongly associated with increased postoperative mortality, morbidity and prolonged hospitalisation. ${ }^{237}$

There are well-reported pathophysiological effects of anaesthesia and upper abdominal surgery on the respiratory system including prolonged lung volume reductions, diaphragm dysfunction, alveolar collapse and reduced mucociliary clearance. ${ }^{8}{ }^{9}$ The combination of which establishes a pathological environment for bacterial growth and impaired pulmonary gas exchange, which can lead to postoperative respiratory failure and/or pneumonia. ${ }^{1011}$

Following surgery, respiratory optimisation and support is warranted to avoid respiratory failure and subsequent reintubation. ${ }^{12}$ 
Conventional low-flow oxygen therapy is commonly administrated via nasal cannula or a face mask to supplement oxygenation yet may not be effective to compensate for loss of lung volume. ${ }^{13}$ While oxygen support alone may be sufficient for low-risk patients in the postoperative period, increased attention to patients at high-risk of PPC development to provide additional therapies that aim to increase postoperative lung volumes may be warranted.

Non-invasive ventilation (NIV) has been shown to reverse reduced lung volumes induced by anaesthesia and abdominal surgery. ${ }^{11}$ During NIV, the positive airway pressure throughout the breath cycle may reopen atelectatic alveoli, increase lung volume and improve gas exchange. ${ }^{11}$ Postoperative NIV has been reported to reduce PPC by half, with a further significant subgroup effect specifically for preventing pneumonia ${ }^{14-17}$ following upper abdominal surgery. While the optimal preventative NIV intervention dosage parameters are currently undetermined, the timing of postoperative NIV initiation is argued to have an important influence on its effectiveness with earlier application of NIV thought to lead to more successful alveolar recruitment. ${ }^{18-20}$ Despite relatively good evidence supporting the use of NIV in the early postoperative period to reduce PPC, the implementation of broad-scale routine prophylactic NIV use is currently unclear but appears to be limited. ${ }^{41}$ The reasons for which are unknown yet likely multifactorial, including perceived risks, resources required and associated service costs. It is possible that newer modalities such as high-flow nasal oxygen therapy could be a viable and more feasible alternative than preventative NIV to reduce PPC.

High-flow nasal oxygen therapy delivers heated and humidified oxygen and/or air via nasal prongs at a prescribed accurate fraction of inspired oxygen $\left(\mathrm{FiO}_{2}\right)$ and with a maximum flow rate of 60 litres per minute. This constant high gas flow at the nares creates a flow-dependent, low level of positive airway pressure between $5 \mathrm{cmH}_{2}$ $\mathrm{O}$ and $8 \mathrm{cmH}_{2} \mathrm{O}^{22}{ }^{23}$ It is hypothesised that this low level of positive pressure increases lung volumes and improves oxygenation $^{24}{ }^{25}$ and may potentially decrease the incidence of respiratory complications post extubation and post surgery. ${ }^{26}$ Compared with standard oxygen therapy, high-flow nasal oxygen therapy reduces reintubation rates and desaturation episodes in critically ill intensive care unit (ICU) patients with acute respiratory failure ${ }^{27}$ and reduces the requirement for escalation of respiratory support following cardiac surgery. ${ }^{28}$ When compared with NIV, high-flow nasal oxygen therapy provided to prevent intubation was superior in reducing 90-day morality in patients with acute respiratory failure in ICU. ${ }^{29}$ Following cardiothoracic surgery, high-flow nasal oxygen therapy demonstrated equivalence with NIV in reducing postsurgery reintubation in patients who developed respiratory failure or were deemed at risk of respiratory failure following postsurgical extubation. ${ }^{30}$

Following major abdominal surgery, it is possible that high-flow nasal oxygen therapy may assist in preventing PPCs. It could be just as effective as NIV and potentially more feasible in terms of resources required and service costs. This has yet to be established as all previous NIV clinical trials ${ }^{14}{ }^{17}$ investigating the prevention of PPC following abdominal surgery have compared NIV wit standard oxygen therapy alone, never to high-flow nasal oxygen therapy. A recent large multicentre randomised control trial (RCT) (Optiflow for prevention of post-extubation hypoxemia after abdominal surgery (OPERA) trial) ${ }^{31}$ demonstrated no benefit in preventing hypoxaemia following major abdominal surgery with the use of preventative high-flow nasal oxygen therapy compared with standard oxygen therapy. Participants were provided with high-flow nasal oxygen therapy postoperatively for a median duration of 15 (IQR 12-18) hours following extubation. ${ }^{31}$ As postoperative respiratory failure commonly occurs within 72 hours after surgery ${ }^{32}$ and functional residual capacity is shown to reach its lowest value 1-2 days following upper abdominal surgery, ${ }^{33-35}$ it may be that high-flow nasal oxygen therapy needs to be prescribed for a longer duration to be clinically effective in preventing PPCs in the postoperative period. It has been recommended that the utility of postoperative high-flow nasal oxygen therapy in high-risk patients when used for longer durations be explored. ${ }^{36}$

Due to the growing exploratory evidence supporting the theoretical and proposed clinical benefits of high-flow nasal oxygen therapy, ${ }^{3738}$ clinical uptake has increased, ${ }^{39}$ and the application of high-flow nasal oxygen therapy is becoming widespread in ICUs ${ }^{40}$ including at our own institution ${ }^{41}$ and also in other clinical settings including the ward. ${ }^{42}$ Given this increasing use of high-flow nasal oxygen therapy yet uncertainty regarding the preventative properties, increased reported patient comfort/tolerance compared with $\mathrm{NIV}^{43}$ and unknown comparative costs of providing a NIV and/or high-flow nasal oxygen therapy service to high-risk upper abdominal surgery patients, this study is designed to detect whether there is a possible signal towards reduction in PPC with the use of intermittent NIV in addition to continuous high-flow oxygen therapy in the first 48 hours after surgery and measure the feasibility of providing these interventions. This study is also designed to understand the associated costs of service delivery for both these therapies. These findings will assist in designing and conducting future multicentre trials.

\section{Pilot work}

Prior to commencing this pilot RCT, we undertook an observational study to test the feasibility and safety of intermittent physiotherapy-led NIV following high-risk elective upper abdominal surgery. ${ }^{41}$ While physiotherapy-led NIV was able to be delivered within 24 hours following surgery and was shown to be safe in both ICU and ward patients, ${ }^{41}$ the main barrier identified to early postoperative NIV was physiotherapy service-related limitations. ${ }^{41}$ Due to lengthy surgeries, a large proportion of patients did not return to the ward or ICU until after our hospital's standard physiotherapy working hours. These patients missed receiving 
the planned initial NIV dose within the target 4 hours. On average, our patients received their first NIV session at 18 hours postsurgery. To mitigate this problem, we implemented a flexible-hour physiotherapy NIV service in the immediate postanaesthesia care unit (PACU), also known as the recovery room. Providing NIV in the PACU has been reported to be feasible and safe. ${ }^{44}$

\section{Objectives}

This project is a pilot RCT with the aim of planning a future definitive multicentre RCT to compare the use of additional intermittent physiotherapy-led NIV to continuous high-flow nasal oxygen therapy alone following elective high-risk upper abdominal surgery to reduce PPC incidence. The primary objective of this pilot study is to detect whether there is a possible signal towards PPC reduction with additional NIV compared with highflow nasal oxygen therapy. Secondary objectives are to measure the feasibility of: (1) trial conduct and design and (2) physiotherapy-led NIV and a high-flow nasal oxygen therapy protocol, safety of NIV therapy and to provide preliminary costs of care information on NIV and high-flow nasal oxygen therapy following upper abdominal surgery. In addition, this trial will explore possible effects on postsurgical ICU and hospital length of stay (LOS); unplanned ICU admission at any time-point during the acute postsurgical stay; incidence of reintubation; in-hospital, 30-day and 12-month all-cause mortality; and health-related quality of life (HRQoL). As this study is a pilot, there is no formal hypothesis.

\section{METHODS \\ Design}

The Non-Invasive Positive airway Pressure therapy to Reduce Postoperative Lung complications following Upper abdominal Surgery (NIPPER PLUS) trial is a prospective, single-centre, assessor blinded, parallel group, pilot, randomised controlled trial, with patients randomly assigned via concealed allocation to either usual care (continuous high-flow nasal oxygen therapy for the first 48 hours after surgery and early standardised mobilisation) or intervention (usual care plus five $30 \mathrm{~min}$ NIV sessions). Figure 1 outlines the schedule of enrolment, interventions and assessments. Randomisation is stratified to planned postsurgical destination (ward or high dependency unit (HDU)/ICU). See figure 2 for a Consolidated Standards of Reporting Trials diagram of the NIPPER PLUS trial and table 1 for an overview of the trial methods and design. The methods are reported in accordance with the Standard Protocol Items; Recommendations for Interventional Trials ${ }^{45}$ guidelines for clinical trials and the Template for Intervention Description and Replication ${ }^{46}$ for reporting of interventions.

\section{Patient and public involvement}

There was no involvement from patients or the public in the development or the design of this trial.

\section{Setting}

The NIPPER PLUS trial is being undertaken at a large regional primary referral publicly funded hospital in Australia.

\section{Participants and enrolment}

All patients having major surgery at our hospital are required to attend a preadmission assessment clinic within 6 weeks of surgery. At this clinic, any patient listed for elective major abdominal surgery receives respiratory physiotherapy education on the prevention of PPC and breathing exercise training. ${ }^{47}$ For the NIPPER PLUS trial, all patients are screened by the preoperative physiotherapist using the Melbourne Risk Prediction Tool (MRPT) ${ }^{6}$ to determine if they are at high risk of developing a PPC. These patients, and any patient with a planned postoperative admission to ICU or HDU, are invited by the preoperative physiotherapist to participate in the trial. Eligible patients are provided with a verbal explanation of the trial and provision of written and pictorial information. Consenting patients are required to sign a written consent form. Where the preoperative physiotherapist or the eligible patient is unable to attend the preadmission clinic, the patient is contacted by telephone and invited to enter the trial. The consent form is then signed during their hospital admission. Participant recruitment began in March 2017 and aims to be completed by August 2018, with final follow-up to be August 2019.

\section{Eligibility criteria}

Inclusions

Eligible participants are patients meeting the following criteria:

1. Adults ( $\geq 18$ years) undergoing elective upper abdominal surgery, able to understand verbal instructions in English and provide informed consent.

2. Open and/or hand-assisted laparoscopic upper abdominal surgery with an abdominal incision longer than $5 \mathrm{~cm}$ that is above or extending above the umbilicus.

3. At high-risk of PPC defined in hierarchal order: (1) a planned postsurgical admission to ICU/HDU and (2) Identified at high-risk using the MRPT. ${ }^{6}$

\section{Exclusions}

The following exclusion criteria apply:

1. Pre-existing obstructive sleep apnoea where overnight continuous positive airway pressure is required.

2. Extreme claustrophobia and inability to tolerate use of a NIV face mask.

3. Current hospital patient for a separate episode of care.

4. Patients requiring oesophageal surgery or organ transplant.

5. Any absolute contraindications for NIV in the period following surgery prior to first NIV session (table 2).

Randomisation and allocation

A research assistant independent to the trial preprepared 130 sequentially numbered (1-130) opaque 


\begin{tabular}{|c|c|c|c|c|c|c|c|c|c|}
\hline & \multirow[b]{2}{*}{ TIMEPOINT } & \multirow{2}{*}{$\begin{array}{c}\text { Enrolment } \\
\text { Listed for } \\
\text { elective } \\
\text { surgery }\end{array}$} & \multicolumn{2}{|c|}{ Allocation } & \multicolumn{3}{|c|}{ Post Allocation } & \multicolumn{2}{|c|}{ Close out } \\
\hline & & & $\begin{array}{c}\text { Pre- } \\
\text { admission } \\
\text { clinic }\end{array}$ & $\begin{array}{l}\text { Day of } \\
\text { surgery }\end{array}$ & $\begin{array}{l}P O D \\
1-7\end{array}$ & $\begin{array}{l}P O D \\
8-14\end{array}$ & $\begin{array}{l}\text { Hospital } \\
\text { D/C }\end{array}$ & $\begin{array}{c}P O D \\
30\end{array}$ & $\begin{array}{c}12 \\
\text { month }\end{array}$ \\
\hline \multirow{4}{*}{ ENROLMENT: } & Enrolment & & & & & & & & \\
\hline & Eligibility screen & $x$ & & & & & & & \\
\hline & Informed consent & & $x$ & & & & & & \\
\hline & Random Allocation & & & $y$ & & & & & \\
\hline \multirow{2}{*}{ INTERVENTIONS: } & $\begin{array}{r}\text { Control; High-flow nasal oxygen } \\
\text { therapy }\end{array}$ & & & $x$ & $x$ & & & & \\
\hline & $\begin{array}{l}\text { Intervention: High-flow nasal } \\
\text { oxygen therapy plus intermittent } \\
\text { postoperative NIV }\end{array}$ & & & $\mathrm{x}$ & $x$ & & & & \\
\hline \multirow{3}{*}{ VARIABLES: } & $\begin{array}{r}\text { Demographics, medical history, } \\
\text { HRQoL }\end{array}$ & & $\mathrm{x}$ & & & & & & \\
\hline & Intraoperative variables & & & & $\mathrm{x}$ & & & & \\
\hline & Postoperative variables & & & & $\mathrm{x}$ & $x$ & $\mathrm{x}$ & & \\
\hline \multirow{7}{*}{ OUTCOMES: } & $P P C$ & & & & $\mathrm{x}$ & $x$ & & & \\
\hline & $\begin{array}{r}\text { Recruitment ability, } \\
\text { physiotherapy-led NIV and high- } \\
\text { flow nasal oxygen therapy } \\
\text { protocol adherence }\end{array}$ & & & & $x$ & & & & \\
\hline & $\begin{array}{r}\text { Major adverse events and/or } \\
\text { transient physiological events of } \\
\text { NIV }\end{array}$ & & & & $\mathrm{x}$ & & & & \\
\hline & $\begin{array}{r}\text { Associated costs of } \\
\text { physiotherapy-led NIV \& a high- } \\
\text { flow nasal oxygen therapy service }\end{array}$ & & & & $x$ & $x$ & $\mathrm{x}$ & & \\
\hline & $\begin{array}{l}\text { Pneumonia, Hospital and ICU } \\
\text { LOS, ICU readmission and } \\
\text { reintubation rates, in-hospital } \\
\text { mortality }\end{array}$ & & & & $x$ & $x$ & $x$ & & \\
\hline & $H R Q \circ L(E Q-5 D-5 L)$ & & & & $x$ & $x$ & & & $x$ \\
\hline & $\begin{array}{r}\text { 30-day and 12-month all-cause } \\
\text { mortality }\end{array}$ & & & & & & & $\mathrm{x}$ & $x$ \\
\hline
\end{tabular}

Figure 1 NIPPER PLUS participant timeline and schedule of events. D/C, discharge; HRQoL, health-related quality of life; ICU, intensive care unit; LOS, length of stay; NIPPER PLUS, Non-Invasive Positive airway Pressure therapy to Reduce Postoperative Lung complications following Upper abdominal Surgery; NIV, non-invasive ventilation; POD, postoperative day; PPC, postoperative pulmonary complication.

envelopes each containing an allocation card wrapped in aluminium foil. Allocation sequence is generated by a web-based computer program (http://www.randomizer. org/). Random allocation is stratified to planned postsurgical destination (ICU and ward). One of the aims of this study is the feasibility of high-flow nasal oxygen therapy and NIV application. The ease of application could be biased towards it being more or less feasible in one location over another. Stratification ensures that there will be equal representation of participants at both locations. At our centre, historical data find that approximately $70 \%$ of high-risk upper abdominal surgery patients have a planned postoperative ICU admission. To manage this difference in location distribution, the total sample size of 130 is divided into two blocks with 90 in the ICU block and 40 in the ward block. The allocation sequence in each block is then determined in a 1:1 ratio, control and intervention. Following construction of the randomisation envelopes, the allocation sequence is locked securely in the hospital's research institute and unavailable to site investigators, those who enrol participants and/or assign interventions.

If it arises that the ratio of eligible ward or ICU patients is different than previously ascertained this will mean that one of the blocks (two blocks stratified to location: ICU or ward) of envelopes will become exhausted prior to completion of the trial. If this occurs, the next available envelope for the other intended postoperative location (ICU or ward), regardless of the actual postoperative location, will be opened in sequence and so on until the minimum target sample of 130 is met. If the situation occurs where the minimum sample is achieved prior to the completion of the funded time period (see sample size section), a block of non-stratified allocation opaque 


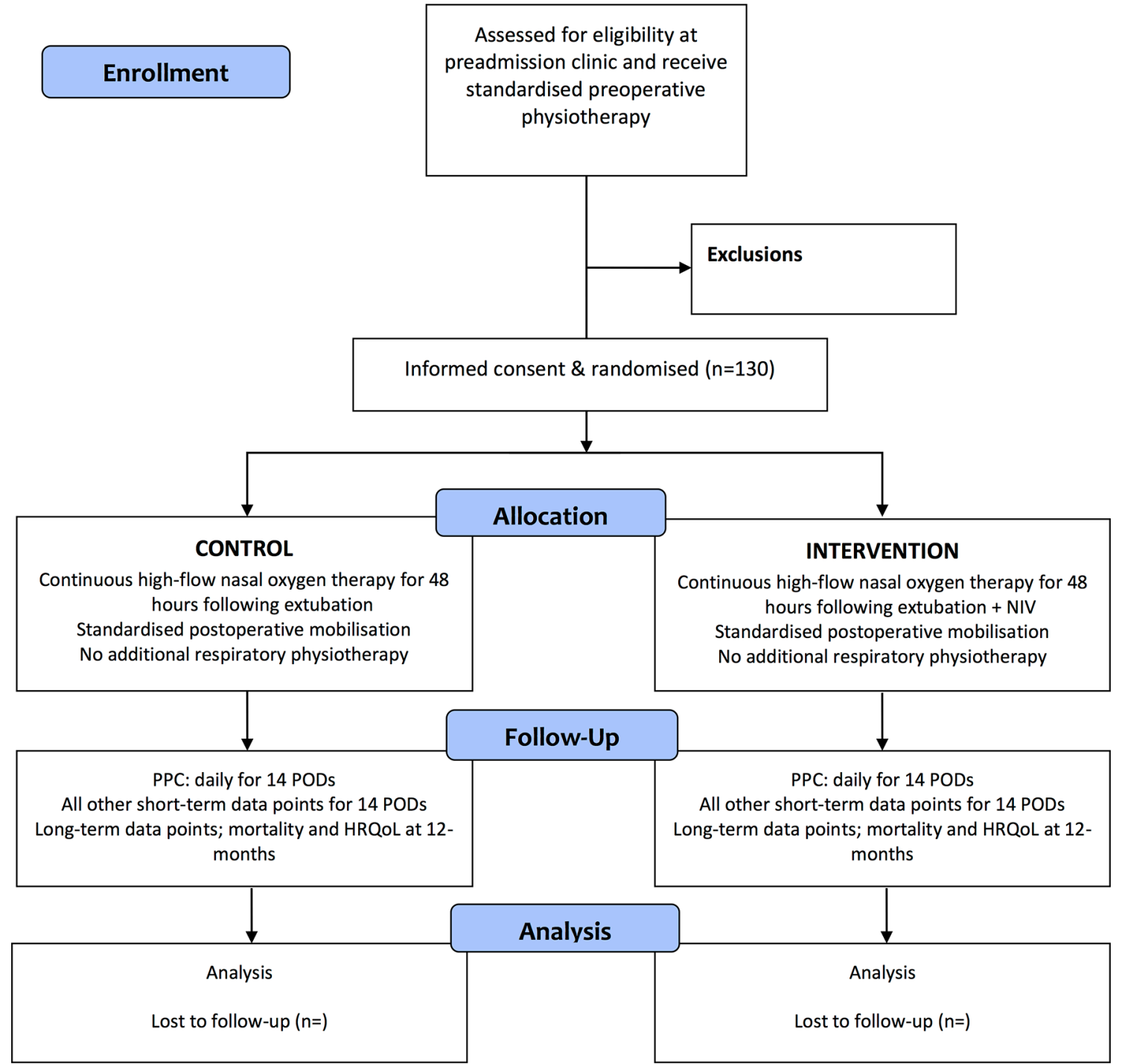

Figure 2 CONSORT flow diagram for the NIPPER PLUS study. CONSORT, Consolidated Standards of Reporting Trials; HRQoL, health-related quality of life; NIPPER PLUS, Non-Invasive Positive airway Pressure therapy to Reduce Postoperative Lung complications following Upper abdominal Surgery; NIV, non-invasive ventilation; POD, postoperative day; PPC, postoperative pulmonary complication.

sealed envelopes will be constructed by an independent administration assistant using the same web-based computer randomisation programme at a $1: 1$ ratio (control:intervention) in a single block of 15, and then repeated as necessary until trial completion.

Entry into the trial is finalised at the end of the surgical procedure where the postsurgical destination is confirmed and exclusion criteria is assessed. Eligible consenting patients are then randomised into the trial by the lead or a site investigator only by opening the next sequentially numbered sealed opaque envelope according to the patient's planned postsurgical destination (ward or ICU/HDU). Once opened, participant's details are written on the envelope to ensure that patients were randomised in presenting order and these are filed securely along with the signed consent form. If a patient is identified as ineligible following surgery completion, they will not be randomised nor entered into the trial.
Participants are randomly assigned to receive either (1) continuous high-flow nasal oxygen therapy for 48 hours following extubation (control group) or (2) continuous high-flow nasal oxygen therapy for 48 hours following extubation plus five 30 min sessions of NIV implemented by a physiotherapist over the first two postoperative days (intervention group).

\section{Interventions}

Control group (usual care)

All participants receive preoperative respiratory physiotherapy education and training. ${ }^{47}$ Postoperatively, an early ambulation programme is provided as per a standardised protocol ${ }^{48}$ of once daily physiotherapy-directed assisted ambulation (table 3). Participants are provided with early ambulation until a threshold score is met using a criteria-lead scoring tool, ${ }^{49}$ or until discharged from hospital, whichever occurs first. If a participant is 
Table 1 Trial registration data set for NIPPER PLUS trial

\section{Data category}

Primary registry and trial identifying number

Date of registration in primary registry

Secondary identifying numbers

Trial protocol version

Source of monetary or material support

Contact for public queries

Contact for scientific queries

Public title

Scientific title

Countries of recruitment

Health condition(s) or problem(s)

studied Intervention(s)

Key inclusion and exclusion criteria

\section{Information}

Australian New Zealand Clinical Trials Registry number: ACTRN12617000269336

\section{$22 / 02 / 2017$}

$\mathrm{n} / \mathrm{a}$

This is version 2 of the protocol and was enacted on February 2017.

Clifford Craig Foundation (\$A80 000)

JL: jane.lockstone@ths.tas.gov.au

JL: jane.lockstone@ths.tas.gov.au

Does early postoperative non-invasive ventilation (NIV) prevent chest infections following high-risk elective abdominal surgery

NIPPER-PLUS trial - Non-Invasive Positive airway Pressure therapy to Reduce Postoperative Lung Complications following Upper abdominal Surgery: a single centre pilot randomised control trial

Australia

Pulmonary complications following high-risk elective upper abdominal surgery.

Active comparator: physiotherapy-led postoperative NIV therapy.

Placebo comparator: high-flow nasal prong oxygen therapy.

Ages edible for study: $\geq 18$ years.

Sexes eligible for study: both.

Accepts health volunteers: no.

Inclusion criteria: all adults undergoing high-risk elective open and/or advanced

hand-assisted laparoscopic abdominal surgery.

Exclusion criteria: (1) any absolute contraindications for NIV in the period following surgery prior to the first NIV session; (2) oesophageal surgery; (3) obstructive sleep apnoea requiring continuous positive airway pressure overnight; (4) extreme claustrophobia; (5) not able to understand verbal instructions in English; (6) do not have capacity to give consent themselves; (7) a current hospital patient for a separate episode of care; and (8) requiring organ transplant.

\begin{tabular}{ll} 
Study type & $\begin{array}{l}\text { Type: investigator initiated, interventional, non-pharmacological, pilot study. } \\
\text { Allocation: concealed randomisation. } \\
\text { Intervention model: parallel assignment. } \\
\text { Masking: assessor blinding. } \\
\text { Primary purpose: prevention. } \\
\text { Phase: phase 2. }\end{array}$ \\
\hline 23/02/2017 \\
\hline Date of first enrolment & Minimum 130 \\
\hline Target sample size & Recruiting \\
\hline Recruitment status & Postoperative pulmonary complication during the first 14 postoperative days. \\
\hline Primary Outcome & $\begin{array}{l}\text { Recruitment ability, physiotherapy-led NIV and high-flow nasal oxygen therapy } \\
\text { protocol adherence, safety of NIV therapy, associated costs of high-flow nasal }\end{array}$ \\
\hline Key secondary outcomes & oxygen therapy and a physiotherapy-led NIV service following upper abdominal \\
& surgery. In addition, this study will explore effects on incidence of pneumonia; \\
intensive care unit (ICU) and hospital length of hospital; ICU readmission rates; \\
incidence of reintubation; in-hospital, 30-day and 12-month all-cause mortality; and \\
health related quality of life.
\end{tabular}

referred for a mobility review, progression of gait aid or a stairs assessment following discharge from physiotherapy, the participant will be treated at the discretion of the ward physiotherapist and this occasion of service recorded. Following surgery, no respiratory physiotherapy is provided to either group unless the participant develops the primary endpoint - a PPC, physiotherapy will then be provided at the discretion of the attending physiotherapist. The type of treatment/s provided will be documented.

On the day of surgery, a site investigator documents high-flow nasal oxygen therapy orders on each consenting patient's postanaesthetic observation chart to instruct theatre nursing staff to initiate high-flow nasal oxygen 


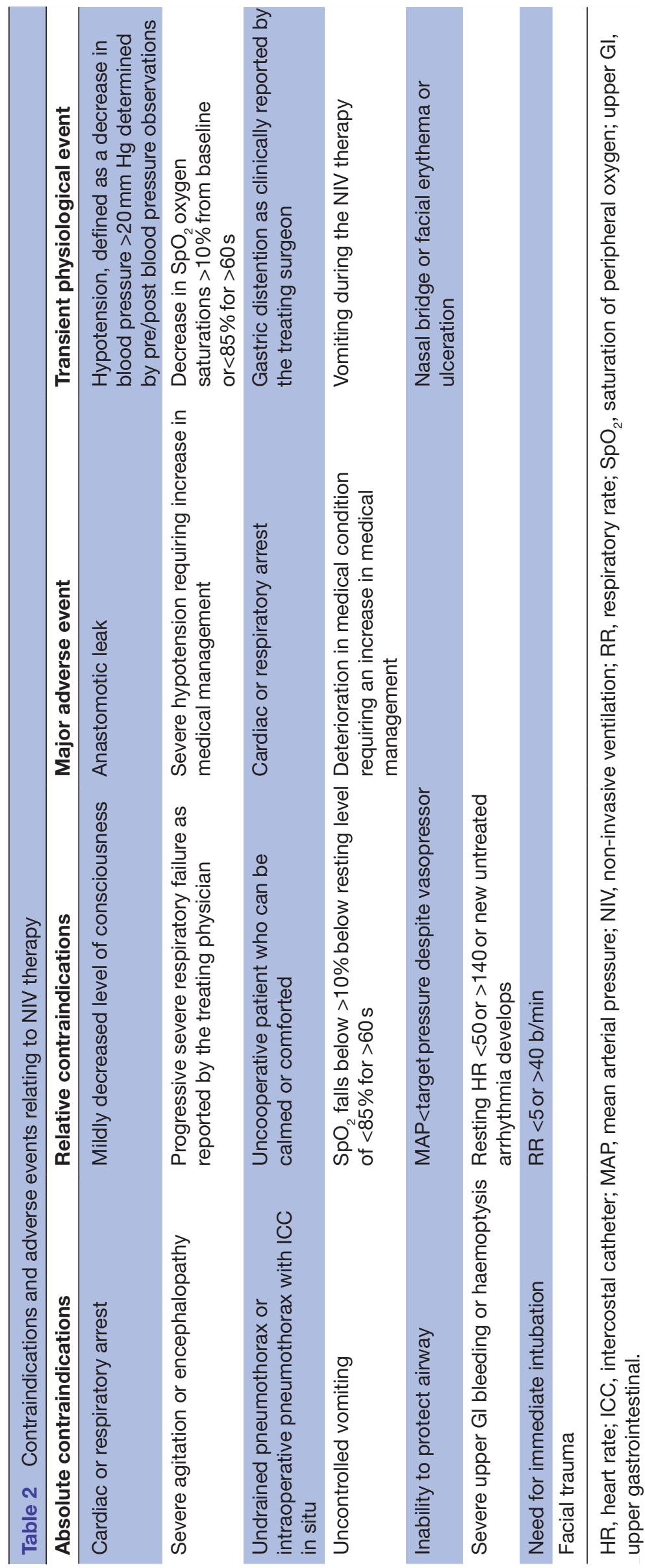

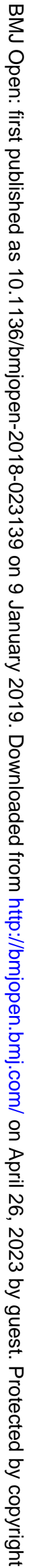




\begin{tabular}{|c|c|}
\hline Stage 1 (safety) & $\begin{array}{l}\text { Sit over edge of bed/sit in chair } \\
\text { minimum of } 2 \mathrm{~min} \text {. }\end{array}$ \\
\hline Stage 2 (safety) & March on spot 0-1 min. \\
\hline Stage 3 (ambulation) & $\begin{array}{l}\text { March on spot/walk away from } \\
\text { bedside } 1-3 \mathrm{~min} .\end{array}$ \\
\hline Stage 4 (ambulation) & $\begin{array}{l}\text { March on spot/walk away from } \\
\text { bedside } 3-6 \mathrm{~min} \text {. }\end{array}$ \\
\hline Stage 5 (ambulation) & Walk away from bedside 6-10 min. \\
\hline Stage 6 (ambulation) & Walk away from bedside $10-15 \mathrm{~min}$. \\
\hline Stage 7 (ambulation) & Walk away from bedside $>15 \mathrm{~min}$. \\
\hline
\end{tabular}

Protocol

Provide assisted early ambulation as soon as possible on the first postoperative day.

At each session progress through each stage in sequence. Time achieved in the session is accumulative.

Aim to achieve rating of perceived exertion of greater than 3/10. Aim to assist patient to ambulate more than $10 \mathrm{~min}$ (stage 6 or greater).

Once patient able to ambulate past stage 3, patient can be assisted to ambulate with a physiotherapy assistant, as long as safe to do as determined by the ward physiotherapist. Interval training is permissible to obtain target walking time. Each interval of rest time must not exceed the preceding work time. Total session time is the accumulative work time.

Provide assisted early ambulation once a day until discharged according to the discharge scoring tool. ${ }^{49}$

therapy as soon as possible following extubation. These orders specify that the $\mathrm{FiO}_{2}$ is to be titrated to achieve a saturation of peripheral oxygen $\left(\mathrm{SpO}_{2}\right)$ between $92 \%$ and $96 \%$ unless otherwise specified by the attending anaesthetist/ICU consultant. Gas flow rate is set at $50 \mathrm{~L}$ per minute. If a participant is unable to tolerate this flow rate, it can be reduced to a minimum of $30 \mathrm{~L}$ per minute. High-flow nasal oxygen therapy is to be provided continuously for 48 hours from the time of extubation. Changes to flow rate and any removal of high-flow nasal oxygen therapy for more than 15 min during the 48 hours period are recorded.

All other aspects of perioperative patient care, including the type of anaesthesia, postoperative analgesia, surgical techniques and postoperative clinical care are provided at the discretion of the anaesthesia and surgical teams and according to routine clinical practice at our centre. Pragmatically, there will be no attempt to standardise perioperative management or intraoperative ventilation strategies for this study. Our hospital is currently not recognised as an enhanced recovery after surgery (ERAS) site; however, some individual anaesthesia and surgical teams within our hospital adhere to ERAS principles.

\section{Intervention group}

Care is provided as per the control group above, with the exception of five $30 \mathrm{~min}^{11} \mathrm{NIV}$ sessions delivered by a physiotherapist over the first two postoperative days in addition to high-flow nasal oxygen therapy. The initial NIV dose is delivered within 4 hours of extubation, followed by twice daily sessions on postoperative days 1 and 2. This service is provided in the PACU, ICU/HDU or the surgical ward depending on the participant's location at the time of NIV delivery.

Prior to commencing each NIV session, all participants are assessed for absolute contraindications for NIV therapy by the treating physiotherapist (table 2). The NIV sessions are delivered using a ResMed VPAP machine (ResMed, Oxfordshire, UK) with a humidified circuit and standard face mask. This is delivered with participants either sitting up in bed with the bed head raised between $45^{\circ}$ and $90^{\circ}$ or with the participant sitting out of bed in a high back chair. Expiratory positive airway pressure (EPAP) is set at $10 \mathrm{cmH}_{2} \mathrm{O} .{ }^{11}$ Inspiratory positive airway pressure (IPAP) is initially set at $15 \mathrm{cmH}_{2} \mathrm{O}$ and adjusted as required to achieve tidal volumes of at least 6-8 mL/ kg. Participants with BMI $>30$ have a starting EPAP set at $12 \mathrm{cmH}_{2} \mathrm{O}$ and a starting IPAP set at $16 \mathrm{cmH}_{2} \mathrm{O}$. Deviations from these planned settings are reported and documented. The difference between IPAP and EPAP (known as pressure support ventilation (PSV)) is maintained at a minimum of $4 \mathrm{cmH}_{2} \mathrm{O}$, and the maximum total pressure (PSV+EPAP) will be no greater than $25 \mathrm{cmH}_{2} \mathrm{O}^{11}$

If a participant is unable to tolerate the set pressures, reassurance is first given to the participant and the following modifications taken in sequential order, until patient tolerance is achieved:

1. Reduce EPAP to $8 \mathrm{cmH}_{2} \mathrm{O}$ (set minimum).

2. Reduce IPAP to $12 \mathrm{cmH}_{2} \mathrm{O}$ (set minimum) in decrements of $1 \mathrm{cmH}_{2} \mathrm{O}$.

If the participant remains unable to tolerate the therapy despite pressure titration and reassurance, cessation of NIV therapy will occur and be reported. Pressure rise time is set at the slowest speed $(900 \mathrm{~ms})$, and the inspiratory trigger is set to the minimum value. Air leaks are managed by fitting the correct size mask carefully using the mask measure guide provided by ResMed with focus on minimising leaks around the nasogastric tube if present. The ResMed VPAP compensates for air leaks up to $40 \mathrm{~L}$ per minute. Above this a 'high-leak' alarm sounds, and the machine is unable to deliver the set pressure. Any high-leak alarm is monitored, recorded and the mask readjusted accordingly. Ideally, the duration of NIV is to be $30 \mathrm{~min}$ of continuous therapy; however, if NIV therapy needs to be temporarily stopped, therapy time will cease and reason will be documented. Once therapy is restarted, timing will recommence. If a participant is unable to continue with NIV therapy within 5 min of temporarily ceasing, the session is terminated and the reason documented. Supplemental oxygen is titrated through the ResMed VPAP as required to achieve $\mathrm{SpO}_{2}$ 92\%-96\% unless otherwise specified by the medical team. During each NIV session, participants have their high-flow nasal oxygen therapy removed for the duration of NIV therapy and replaced once therapy is finished. The treating physiotherapist continuously monitors all participants for the duration of the NIV therapy and reassesses 30 min postintervention. Data including blood pressure, 
heart rate, respiratory rate and $\mathrm{SpO}_{2}$ are recorded before, immediately after and $30 \mathrm{~min}$ after each NIV session. Any reason resulting in early cessation of NIV intervention or being unable to provide NIV therapy is reported.

All physiotherapists providing the intervention attend NIV training with the ICU senior physiotherapist who has 11 years' experience in NIV application. The training session includes familiarisation with the ResMed VPAP machine, setup of equipment, detailed explanation of the intervention protocol and troubleshooting. The physiotherapists are provided with a training manual and a copy of this manual is also kept with the ResMed VPAP to allow reference at any point during the intervention. The training manual consists of all the information provided in the training session. The years of hospital experience of each participating physiotherapist is reported.

\section{Withdrawal from trial}

Participants are withdrawn for: (1) requiring longer than 48 hours of mechanical ventilation following surgery, or (2) withdrawal of consent. All withdrawals are reported.

\section{Outcomes}

To detect a possible signal towards PPC reduction with the use of NIV in addition to continuous high-flow oxygen therapy in the first 48 hours after surgery, the primary outcome measure is the development of a PPC within the first 14 postoperative days or hospital discharge whichever occurs first. Using the Melbourne Group Scale (MGS) diagnostic Tool Version $2^{48}$ (box 1), a PPC is diagnosed when four or more of eight screening criteria are present in a 24-hour day. The MGS tool is valid and reliable, ${ }^{51}$ is sensitive to therapeutic interventions designed to ameliorate postoperative atelectasis and alveolar derecruitment ${ }^{48}$ and widely utilised in upper abdominal surgery

Box 1 Postoperative pulmonary complications diagnostic tool: Melbourne group scale version $2^{48}$

Diagnosis confirmed when four or more of the following criteria are present anytime in the 24-hour period 00:01 to 24:00 on a single postoperative day:

1. New abnormal breath sounds on auscultation different to preoperative assessment.

2. Productive of yellow or green sputum different to preoperative assessment.

3. Pulse oximetry oxygen saturation $\left(\mathrm{SpO}_{2}\right)<90 \%$ on room air on more than one consecutive postoperative day.

4. Raised maximum oral temperature $>38^{\circ} \mathrm{C}$ on more than one consecutive day.

5. An unexplained white cell count greater than $11 \times 10^{9} / \mathrm{L}$.

6. Presence of infection on sputum culture report.

7. Chest radiograph (CXR) report of collapse/consolidation. When a CXR has been taken but no report available, a ward medical officer or a senior respiratory physiotherapist with more than 10 years' experience will be asked to report.

8. Physician's diagnosis of pneumonia, lower or upper respiratory tract infection, an undefined chest infection or prescription of an antibiotic for a respiratory infection. trials. ${ }^{4-6} 48$ An assessor blinded to group allocation, who has no clinical involvement with the study, assesses participants prospectively and daily for a PPC until the seventh postoperative day. Thereafter, additional PPC assessments are only performed if clinically indicated when there are signs of respiratory deterioration reported in the medical record until postoperative day 14 or hospital discharge, whichever occurs first. To reduce the potential for missing data, retrospective collection of PPC data from the daily medical record will occur when a participant or assessor is unavailable for PPC assessment. Participants scoring three out of the possible eight factors are assessed twice daily to monitor for any further clinical deterioration. A blinded senior physiotherapist confirms a positive diagnosis of a PPC.

\section{Feasibility measures of trial conduct, design and protocol}

1. Consent and recruitment ability. Consent rate is anticipated to be $\geq 90 \%$ with recruitment of one to two patients per week.

2. Protocol adherence of physiotherapy-led NIV therapy. Successful physiotherapy-led NIV implementation is set at $\leq 20 \%$ protocol deviations. This is measured and reported by:

a. Proportion of intervention participants who receive the first NIV session within 4 hours of surgical extubation.

b. Proportion of intervention participants who receive five 30 min NIV sessions in the first two postoperative days.

c. Reasons why NIV therapy could not be delivered or were ceased early.

3. Protocol adherence of high-flow nasal oxygen therapy. Successful high-flow nasal oxygen therapy implementation is set at $<20 \%$ protocol deviations. This is measured and reported by;

a. Proportion of participants who receive high-flow nasal oxygen therapy for 48 continuous hours following surgical extubation.

b. Time in minutes from extubation following surgery to commencement of high-flow nasal oxygen therapy.

c. Reasons why high-flow nasal oxygen therapy cannot be delivered or sustained.

4. Safety of NIV therapy measured by: (1) major adverse events relating to NIV therapy defined as: anastomotic leak suspected and confirmed; severe hypotension requiring an increase in medical management; cardiac and/or respiratory arrest; deterioration in medical condition requiring an increase in medical management and (2) any transient physiological events during or immediately following NIV intervention (table 2).

5. Costs of a high-flow nasal oxygen therapy and physiotherapy-led NIV therapy service measured by: costs of equipment (NIV masks, high-flow and NIV circuits, cleaning and machine service costs); physiotherapy time (in hours) attributed to delivering the NIV 
therapy and costs of an ICU; and hospital stay measured by average cost of a bed day.

\section{Secondary exploratory outcomes}

1. Incidence of pneumonia ${ }^{52}$ defined as new chest radiograph infiltrates with at least two of: temperature $>38^{\circ} \mathrm{C}$, dyspnoea, cough and purulent sputum, altered respiratory auscultation and white cell count (WCC) $>12000 / \mathrm{mL}$ or leucopaenia $<3000 / \mathrm{mL}$ ), within the first 14 postoperative hospital days or hospital discharge whichever occurs first.

2. Incidence of systemic inflammatory response syndrome (SIRS) as defined by two or more of the following: temperature $>38$ or $<36$; heart rate $(\mathrm{HR})>90$; respiratory rate $(\mathrm{RR})>20$ or Partial pressure of carbon dioxide in arterial blood $\left(\mathrm{PaCO}_{2}\right)<32$, or ventilation for acute process; WCC $>12$ or $<4$, within the first 14 postoperative hospital days or hospital discharge, whichever occurs first.

3. Incidence of sepsis, defined as a Sequential Organ Failure Assessment score $\geq 2$, within the first 14 postoperative hospital days or hospital discharge, whichever occurs first.

4. Postsurgical ICU and hospital LOS in days.

5. Unplanned ICU admission at any time-point during the acute postsurgical stay.

6. Incidence of reintubation at any time-point during the acute postsurgical stay.

7. In-hospital, 30-day and 12-month mortality.

8. HRQoL using the EQ-5D-5L ${ }^{53}$ preoperatively, postoperative day 7 and day 14 and at 12 months postoperatively.

\section{Blinding}

Random allocation occurs following completion of surgery. This ensures preadmission and operating theatre medical, nursing and physiotherapy staff are masked to postoperative group assignment. Postoperatively, PPC assessors are independent of routine postoperative clinical care and masked to group allocation. All physiotherapy documentations relating to the NIV intervention are documented and filed separately to ensure PPC assessors remain blinded for the first seven postoperative days and then added to the patient's medical file. If a treatment group participant informs the PPC assessor of their group allocation, this is noted and reported. Due to the nature of intervention, postoperative ward staff including nurses, doctors and treating physiotherapists are unable to be blinded.

\section{Data collection}

Preoperative variables

To measure baseline characteristics, the following variables are collected directly from the participant or the medical record: age (years), gender, height $(\mathrm{cm})$, weight $(\mathrm{kg})$, body mass index $\left(\mathrm{kg} / \mathrm{cm}^{2}\right)$, planned surgical procedure, surgical category and reason for the procedure, physical health status according to the American Society of Anaesthesiologists and rated by the attending anaesthetist at the pre-admission clinic (PAC) (score 1-5), smoking history (non-smoker, current smoker or ex-smoker having ceased more than 8 weeks preoperatively), smoking pack years (one pack year $=20$ cigarettes per day for 1 year), years since smoking cessation, respiratory status including auscultation signs and $\mathrm{SpO}_{2}(\%)$ on room air, cough strength and presence of sputum, participant-reported history of a chest infection in the previous 2 weeks, functional comorbidity index, ${ }^{54}$ participant-reported estimated maximum metabolic equivalent physical activity using a self-rated physical Specific Activity Questionnaire ${ }^{55}$ and any limiting factor to ambulation.

\section{Intraoperative variables}

The following variables are collected from the anaesthetic record, operation report and medical record: duration of anaesthesia (in minutes) during surgery; mechanical ventilation parameters including mode of ventilation, level of pressure/volume control, positive end expiratory pressure used and any recruitment manoeuvres performed; average $\mathrm{FiO}_{2}$ during surgery; type and amount of intraoperative fluid delivered $(\mathrm{mL} / \mathrm{kg} /$ hour $)$; number and type of blood transfusion units; and incision type.

\section{Postoperative variables}

Postoperative data are collected daily for the first 14 days or until hospital discharge, whichever occurs first for the following variables: time in days from the preoperative physiotherapy session to the operation; location (ICU or surgical ward) and duration in days at each location; duration of analgesia and type (epidural, constant opioid infusion, patient controlled analgesia, patient controlled epidural analgesia, oral, local pain infusion or other); unplanned ICU admission and ICU LOS; hospital LOS; hours of mechanical ventilation; days of vasopressor use; days and types of oxygen therapy use; total days of nasogastric tube; and day and diagnosis of a prolonged postoperative ileus using a standardised criteria ${ }^{56}$ of two or more of the following factors in a 24-hour period including nausea/vomiting, inability to tolerate normal diet, absence of flatus, abdominal distension, radiologic confirmation and physician diagnosis of ileus. Postoperative NIV parameters are collected including: time in hours from extubation following surgery to the first NIV session; time each NIV session is delivered and the grade/seniority of the treating physiotherapist providing the NIV; position of the patient during NIV; duration in minutes of each NIV session; IPAP and EPAP used; pressure titration-reasons if pressure titration occurs and the pressures used; number of times NIV has to temporarily ceased prior to the planned $30 \mathrm{~min}$ session; reasons NIV was unable to be delivered to the participant; and any major adverse or transient physiological event that occurs as a direct result of NIV therapy. Postoperative high-flow nasal oxygen therapy parameters are collected including: time in minutes from extubation following surgery onto high-flow nasal oxygen therapy; time and date high-flow nasal oxygen therapy is removed; duration in hours of 
high-flow nasal oxygen therapy within the first 48 postoperative hours; number of times high-flow nasal oxygen therapy is removed for greater than $15 \mathrm{~min}$ within the first two postoperative days; average flow rate during the first two postoperative days; average $\mathrm{FiO}_{2}$ during the first two postoperative days; and reasons a participant is unable to have postoperative high-flow nasal oxygen therapy for the first two postoperative days. Early ambulation parameters are collected including: time in hours from end of surgery until time to ambulation $>1$ min; postoperative day walked longer than $10 \mathrm{~min}$; maximum rating of perceived exertion during ambulation at each session; and maximum ambulation stage attained at each session and reasons for a participant being unable to participate in an ambulation session.

\section{Sample size}

This RCT is a pilot trial that has been funded to be conducted for a defined time period (18 months). Current surgical throughput of eligible patients at our hospital predicts that we will recruit a sample of 130 eligible participants (65 per group) in the trial period. If this sample is not reached within the funded time period, recruitment will continue until a minimum sample of 130 is met. If this sample is reached prior to the designated funding period (18 months), recruitment will continue past 130, until this time period is completed. A baseline PPC rate of $18 \%$ for the control group (high-flow nasal oxygen therapy alone) is anticipated based on historical Lung Infection Prevention Post Surgery - Major Abdominal - with Pre-Operative Physiotherapy trial (LIPPSMAck POP $)^{48}$ data $(n=101)$ of matched high-risk elective upper abdominal surgery participants who were given the same standardised preoperative and postoperative physiotherapy as planned in NIPPER PLUS.

Previous systematic reviews in NIV to prevent pneumonia following surgery report a relative risk reduction of approximately $60 \% .{ }^{1457}$ Using inference for proportion calculations for two independent samples, a total sample of 130 (two groups of 65) would detect a $50 \%$ relative risk reduction in PPC between groups (favouring the NIV group, one-sided alpha at 0.05 ) with only $44 \%$ power. This sample will only be adequately powered $(80 \%)$ if there is a large $75 \%$ relative risk reduction in PPC with the application of NIV (18\% down to $4 \%$ ).

Assuming that NIV is superior to high-flow nasal oxygen therapy, an adequately powered study would need a sample of at least 450 (relative risk reduction $50 \%$ from a baseline of $18 \%$, alpha two-sided 0.05 , beta $80 \%$ ) which would require a multicentre approach. However, there is also the possibility that high-flow nasal oxygen therapy is just as effective as NIV to prevent PPC. This would require a non-inferiority trial and would require a much larger sample.

This pilot study aims to measure the feasibility of the intervention protocol and provide a baseline estimate of effect to assist in determining the design (superiority or non-inferiority) and conduct of a future multicentre RCT.

\section{Methods: data collection, management and analysis}

Data are collected from participants using a standardised electronic case report form and stored on a password-protected electronic hard drive. Research assistants and site investigators responsible for data collection are trained directly by the principal investigator to ensure correct data handling. Any data or participant lost to follow-up will be reported. Once each participant's data set is completed, it is deidentified, entered into a main database, locked and maintained securely by the principal investigator. All data, consent forms and relevant correspondence are stored according to Australian privacy laws and archived for a minimum of 12 years. On completion of the trial, the database will be made available for independent analysis or as an appendix in the publishing journal if requested.

\section{Statistical methods}

As our study is stratified to postoperative location (ICU/ ward) only, there is a possibility of significant baseline differences between groups. This will be managed according to the prognostic strength and size of imbalances due to potential confounding baseline variables between groups being assessed. ${ }^{58}$ Adjustment covariates will be selected by backward stepwise regression from covariates that may have the potential for clinically significant alterations in effect sizes. These include: smoking history, age, length in time of operation, operation category (upper gastrointestinal, colorectal, urological and other), incision type and location, ${ }^{59}$ intraoperative ventilation strategies, ${ }^{360}$ fluid delivery, ${ }^{61}$ blood transfusions ${ }^{62}$ and mode of postoperative analgesia. ${ }^{63}$

The primary outcomes of absolute and relative rates of PPC in the trial groups will be estimated using multivariate robust random effects Poisson generalised linear modelling to allow assessment of binary outcomes with or without adjustment for potential confounding variables (incidence rates and rate ratios, 95\% CIs and $p$ values). In addition, the effect of time from the end of surgery/ anaesthesia to diagnosis of PPC will be compared using Cox proportional hazards regression with and without covariate adjustment (HR, 95\% CIs and $\mathrm{p}$ values). Graphic representation of this analysis will be performed using the Kaplan-Meier method.

Although this study is not adequately powered, a number of secondary outcomes will be treated as time-toevent analyses, with HRs estimated using Cox proportional hazards regression: (1) the day of first diagnosis of other events will be recorded (pneumonia, SIRS, sepsis, reintubation and death) and (2) treatment group comparison for time from surgery to readiness for discharge, and to actual discharge (LOS), will be made using Cox proportional hazards regression, with successful discharge treated as censoring 'failure' and death or no discharge within 30 days treated as censoring 'non-failure'. Binomial secondary outcomes including unplanned ICU admission, unplanned reintubation will be analysed using mixed effects Poisson regression. Secondary outcomes with irregular distributions, including length 
of time periods (ICU and total postoperative LOS) and HRQoL, will be evaluated for group differences using mixed effects ordered logistic regression, with mean time (95\% CI) estimated for descriptive purposes using mixed effects linear regression. An intention-to-protocol sensitivity analysis will be performed by excluding from the analysis any participant who did not undergo the planned postoperative NIV intervention treatment. The sensitivity of the outcome estimates to missing data will be evaluated using multiple imputation. All analyses will be performed using Stata V.14 or later and analysed on an intention-totreat basis.

\section{Methods: monitoring \\ Data monitoring}

The steering committee consists of the principal investigator, local investigator and two academic supervisors who contribute to the design and revision of this study protocol. The principal and local investigators are responsible for the study administrative management and daily coordination of the trial ensuring appropriate trial conduct, record keeping and data management.

An independent Data and Safety Monitoring Board (DSMB) monitors the ethics of the study in accordance with the Declaration of Helsinki overseeing safety and conduct of the study.

For the trial, there is a stopping rule for the potential of NIV or high-flow nasal oxygen therapy to be harmful. An unacceptable rate of anastomotic leakage of over $2.5 \%$ will trigger consideration for trial termination by the independent DSMB established for the oversight of this clinical trial. To detect a $2.5 \%$ anastomotic leakage rate in either group requires a minimum of 57 patients (one-sample test of proportion compared with hypothetical $0.1 \%$ rate; power $80 \%$; alpha 0.05 ). Analysis of anastomotic leakage rates only in both groups will therefore be performed at participant recruitment number 60 using cumulative summation analysis. ${ }^{64}$

Any other major adverse events directly relating to the interventions will be reported with oversight from the independent DSMB.

\section{Ethics and dissemination}

Trial results will be disseminated widely through conference presentations and peer-review journal publications.

\section{DISCUSSION}

Consequences of PPCs following upper abdominal surgery are well defined, leading to great interest in their prevention. High-risk patients have been shown to be over eight times more likely to develop a PPC compared with individuals identified as low-risk ${ }^{6}$ suggesting increased attention is required to improve postoperative outcomes in this high-risk cohort.

While previous clinical studies support the use of preventative NIV therapy following major abdominal surgery, ${ }^{11} 14$ implementation of NIV therapy does not appear to be standard postoperative care ${ }^{421}$ and a number of important methodological limitations exists in previous literature including high-bias risk and minimal reporting of adverse events. ${ }^{14}$ Recommendations for future research include; evaluating the use of NIV in preventing mortality, a targeted approach investigating patients at higher risk for PPCs and must report on all adverse effects and possible complications of preventative postoperative NIV. ${ }^{14} 17$ The NIPPER PLUS study is designed to begin targeting these recommendations by collecting and reporting on in-hospital, 30-day and 12-month all-cause mortality for all participants and is recruiting participants identified as high-risk of developing a PPC only. High risk for this study has been defined as either: eligible patients with a planned postoperative admission to ICU/HDU due to this factor being independently associated with the development of a PPC $^{6}$ or eligible patients identified at high risk using the MRPT. ${ }^{6}$ The MRPT has been shown to be specific and sensitive in the identification of individuals who are at highest risk of PPC development in the surgical settings including upper abdominal surgery. ${ }^{56}$

Preventative NIV was associated with no major complications in our observational study, ${ }^{41}$ and the NIPPER PLUS trial aims to further support this finding by reporting on any adverse event as well as transient physiological events directly relating to NIV therapy during, immediately following and 30 minutes after therapy, therefore contributing to necessary and strongly recommended NIV safety data for both ICU and ward patients.

All previous preventative NIV clinical trials in abdominal surgery compare NIV with standard oxygen therapy only $^{14}{ }^{17}$; however, the application of high-flow nasal oxygen therapy is becoming widespread in ICUs ${ }^{40}$ and in other clinical settings. ${ }^{26}$ The NIPPER PLUS study is designed with high-flow nasal oxygen therapy as standard care to match current clinical practice within our ICU unit and aims to detect whether there is a possible signal towards PPC reduction with the use of additional intermittent NIV compared with continuous high-flow oxygen therapy alone. The NIPPER PLUS trial is a single-centre study. The effect of high-flow nasal oxygen therapy in high-risk upper abdominal surgery patients is currently unclear. Prior to undertaking expensive fully powered multicentre trials, there is a need to build evidence and data from pilot trials for realistic effect size variability estimation and to measure the design, feasibility, safety and potential challenges of treatment protocols. This pilot study aims to inform future definitive trial design and conduct. Interpretation of results will be evaluated in context of the studies' limitations and indeed it may be demonstrated that this protocol is unfeasible in its current form and would be futile to progress to multicentre trials without study and protocol redesign.

In conclusion, the NIPPER PLUS trial is a single-centre, assessor-blinded, parallel group, pilot RCT, which aims to detect whether there is a possible signal towards PPC reduction with the use of additional intermittent NIV compared with continuous high-flow oxygen therapy 
alone following high-risk elective upper abdominal surgery. This trial is measuring recruitment ability, feasibility of implementing a physiotherapy-led NIV and highflow nasal oxygen therapy protocol, safety of NIV therapy and preliminary costs of care information on a NIV and high-flow nasal oxygen therapy service. This will assist in the design and conduct of future multicentre trials. In addition, this trial will also explore possible effects on postsurgical ICU and hospital LOS, unplanned ICU admission, reintubation rates, in-hospital, 30-day and 12-month mortality. This trial standardises preoperative and postoperative physiotherapy care and is currently recruiting.

\section{Author affiliations}

${ }^{1}$ Department of Physiotherapy, Launceston General Hospital, Launceston, Tasmania, Australia

${ }^{2}$ Department of Physiotherapy, The University of Melbourne, Melbourne, Victoria, Australia

${ }^{3}$ School of Health Sciences, University of Tasmania, Launceston, Tasmania, Australia ${ }^{4}$ Anaesthesia Perioperative and Pain Medicine Unit, Melbourne Medical School, The University of Melbourne, Melbourne, Victoria, Australia

Acknowledgements We would like to thank all staff in the intensive care unit, the surgical ward, the post anaesthesia care unit and preadmission clinic at Launceston General Hospital; all physiotherapists providing the interventions and the NIPPER PLUS research assistant, Bronte Biggins-Tosch, for her dedication and thoroughness. Lastly, we would like to thank all the participants on their assistance in contributing to the body of knowledge in this area.

Contributors IB and $\mathrm{JL}$ conceived and designed the study and coordinated the trial. LD and SMP assisted in final study design and protocol. JL prepared the first draft of the protocol manuscript and was responsible for the final manuscript. All authors revised all manuscript drafts, approved the final manuscript and contributed intellectually important content. $\mathrm{JL}$ is the guarantor of the paper and takes responsibility for the integrity of the work as a whole, from inception to published article.

Funding This work was supported by the Clifford Craig Foundation, Launceston, Australia (Project 160)

Disclaimer The funding body has no influence on the study design, collection or data analysis or publication of results.

Competing interests None declared.

Patient consent for publication Not required

Ethics approval The trial is being conducted in accordance with the Declaration of Helsinki and has undergone ethics review by the Tasmanian Health Human Research Ethics Committee and received approval 08/02/2017 (protocol reference H0016207). All participants will provide written informed consent.

Provenance and peer review Not commissioned; externally peer reviewed.

Open access This is an open access article distributed in accordance with the Creative Commons Attribution Non Commercial (CC BY-NC 4.0) license, which permits others to distribute, remix, adapt, build upon this work non-commercially, and license their derivative works on different terms, provided the original work is properly cited, appropriate credit is given, any changes made indicated, and the use is non-commercial. See: http://creativecommons.org/licenses/by-nc/4.0/.

\section{REFERENCES}

1. Schultz MJ, Hemmes SN, Neto AS, et al. Epidemiology, practice of ventilation and outcome for patients at increased risk of postoperative pulmonary complications: LAS VEGAS - an observational study in 29 countries. Eur J Anaesthesiol 2017;34:492-507.

2. Serpa Neto A, Hemmes SN, Barbas CS, et al. Incidence of mortality and morbidity related to postoperative lung injury in patients who have undergone abdominal or thoracic surgery: a systematic review and meta-analysis. Lancet Respir Med 2014;2:1007-15.
3. Hemmes SN, Gama de Abreu M, Pelosi P, et al. High versus low positive end-expiratory pressure during general anaesthesia for open abdominal surgery (PROVHILO trial): a multicentre randomised controlled trial. Lancet 2014:384:495-503.

4. Haines KJ, Skinner EH, Berney S, et al. Association of postoperative pulmonary complications with delayed mobilisation following major abdominal surgery: an observational cohort study. Physiotherapy 2013;99:119-25.

5. Parry S, Denehy L, Berney S, et al. Clinical application of the Melbourne risk prediction tool in a high-risk upper abdominal surgical population: an observational cohort study. Physiotherapy 2014;100:47-53.

6. Scholes RL, Browning L, Sztendur EM, et al. Duration of anaesthesia, type of surgery, respiratory co-morbidity, predicted VO2max and smoking predict postoperative pulmonary complications after upper abdominal surgery: an observational study. Aust $J$ Physiother 2009;55:191-8.

7. Fernandez-Bustamante A, Frendl G, Sprung J, et al. Postoperative pulmonary complications, early mortality, and hospital stay following noncardiothoracic surgery: a multicenter study by the perioperative research network investigators. JAMA Surg 2017;152:157-66.

8. Miskovic A, Lumb AB. Postoperative pulmonary complications. Br J Anaesth 2017;118:317-34.

9. Warner DO. Preventing postoperative pulmonary complications: the role of the anesthesiologist. Anesthesiology 2000;92:1467-72.

10. van Kaam AH, Lachmann RA, Herting E, et al. Reducing atelectasis attenuates bacterial growth and translocation in experimental pneumonia. Am J Respir Crit Care Med 2004;169:1046-53.

11. Jaber S, Chanques G, Jung B. Postoperative noninvasive ventilation. Anesthesiology 2010;112:453-61.

12. Ruscic KJ, Grabitz SD, Rudolph MI, et al. Prevention of respiratory complications of the surgical patient: actionable plan for continued process improvement. Curr Opin Anaesthesiol 2017;30:399-408.

13. Futier E, Jaber S. High-flow nasal cannula following extubation: is more oxygen flow useful after surgery? Intensive Care Med 2015;41:1310-3.

14. Ireland CJ, Chapman TM, Mathew SF, et al. Continuous positive airway pressure (CPAP) during the postoperative period for prevention of postoperative morbidity and mortality following major abdominal surgery. Cochrane Database Syst Rev 2014;8:CD008930.

15. Ferreyra GP, Baussano I, Squadrone V, et al. Continuous positive airway pressure for treatment of respiratory complications after abdominal surgery: a systematic review and meta-analysis. Ann Surg 2008;247:617-26.

16. Jaber $\mathrm{S}$, Lescot $\mathrm{T}$, Futier $\mathrm{E}$, et al. Effect of noninvasive ventilation on tracheal reintubation among patients with hypoxemic respiratory failure following abdominal surgery: a randomized clinical trial. JAMA 2016;315:1345-53.

17. Chiumello D, Chevallard G, Gregoretti C. Non-invasive ventilation in postoperative patients: a systematic review. Intensive Care Med 2011;37:918-29.

18. Squadrone V, Coha M, Cerutti E, et al. Continuous positive airway pressure for treatment of postoperative hypoxemia: a randomized controlled trial. JAMA 2005;293:589-95.

19. Ricksten SE, Bengtsson A, Soderberg C, et al. Effects of periodic positive airway pressure by mask on postoperative pulmonary function. Chest 1986;89:774-81.

20. Lindner KH, Lotz P, Ahnefeld FW. Continuous positive airway pressure effect on functional residual capacity, vital capacity and its subdivisions. Chest 1987;92:66-70.

21. Patman S, Bartley A, Ferraz A, et al. Physiotherapy in upper abdominal surgery - what is current practice in Australia? Arch Physiother 2017;7:11.

22. Parke R, McGuinness S, Eccleston M. Nasal high-flow therapy delivers low level positive airway pressure. $\mathrm{Br} \mathrm{J}$ Anaesth 2009;103:886-90.

23. Groves N, Tobin A. High flow nasal oxygen generates positive airway pressure in adult volunteers. Aust Crit Care 2007;20:126-31.

24. Corley A, Caruana LR, Barnett AG, et al. Oxygen delivery through high-flow nasal cannulae increase end-expiratory lung volume and reduce respiratory rate in post-cardiac surgical patients. $\mathrm{Br} J$ Anaesth 2011;107:998-1004.

25. Sztrymf B, Messika J, Bertrand F, et al. Beneficial effects of humidified high flow nasal oxygen in critical care patients: a prospective pilot study. Intensive Care Med 2011;37:1780-6.

26. Ashraf-Kashani N, Kumar R. High-flow nasal oxygen therapy. BJA Educ 2017;17:63-7.

27. Maggiore SM, Idone FA, Vaschetto R, et al. Nasal high-flow versus Venturi mask oxygen therapy after extubation. Effects on oxygenation, comfort, and clinical outcome. Am J Respir Crit Care Med 2014:190:282-8. 
28. Parke R, McGuinness S, Dixon R, et al. Open-label, phase II study of routine high-flow nasal oxygen therapy in cardiac surgical patients. Br J Anaesth 2013;111:925-31.

29. Frat JP, Thille AW, Mercat A, et al. High-flow oxygen through nasal cannula in acute hypoxemic respiratory failure. $N$ Engl $J$ Med 2015;372:2185-96.

30. Stéphan F, Barrucand B, Petit P, et al. High-flow nasal oxygen vs noninvasive positive airway pressure in hypoxemic patients after cardiothoracic surgery: a randomized clinical trial. JAMA 2015;313:2331-9.

31. Futier E, Paugam-Burtz C, Godet T, et al. Effect of early postextubation high-flow nasal cannula vs conventional oxygen therapy on hypoxaemia in patients after major abdominal surgery: a French multicentre randomised controlled trial (OPERA). Intensive Care Med 2016;42:1888-98.

32. Ramachandran SK, Nafiu OO, Ghaferi A, et al. Independent predictors and outcomes of unanticipated early postoperative tracheal intubation after nonemergent, noncardiac surgery. Anesthesiology 2011;115:44-53.

33. Craig DB. Postoperative recovery of pulmonary function. Anesth Analg 1981;60:46-52.

34. Meyers JR, Lembeck L, O'Kane $\mathrm{H}$, et al. Changes in functional residual capacity of the lung after operation. Arch Surg 1975;110:576-83.

35. Denehy L, Carroll S, Ntoumenopoulos G, et al. A randomized controlled trial comparing periodic mask CPAP with physiotherapy after abdominal surgery. Physiother Res Int 2001;6:236-50.

36. Clarke A. OPERA. The bottom Line. $2017 \mathrm{http}: / /$ www.thebottomline. org.uk/summaries/icm/opera

37. Papazian L, Corley A, Hess D, et al. Use of high-flow nasal cannula oxygenation in ICU adults: a narrative review. Intensive Care Med 2016;42:1336-49.

38. Spoletini G, Alotaibi M, Blasi F, et al. Heated humidified highflow nasal oxygen in adults: mechanisms of action and clinical implications. Chest 2015;148:253-61.

39. Lee JH, Rehder KJ, Williford L, et al. Use of high flow nasal cannula in critically ill infants, children, and adults: a critical review of the literature. Intensive Care Med 2013;39:247-57.

40. Renda T, Corrado A, Iskandar G, et al. High-flow nasal oxygen therapy in intensive care and anaesthesia. $\mathrm{Br} J$ Anaesth 2018;120:18-27.

41. Lockstone J, Parry SM, Denehy L, et al. Physiotherapist administered, non-invasive ventilation to reduce postoperative pulmonary complications in high-risk patients following elective upper abdominal surgery; a before-and-after cohort implementation study. Physiotherapy 2018.

42. Millette $\mathrm{BH}$, Athanassoglou $\mathrm{V}$, Patel A. High flow nasal oxygen therapy in adult anaesthesia. Trends in Anaesthesia and Critical Care 2018;18:29-33

43. Huang HW, Sun XM, Shi ZH, et al. Effect of high-flow nasal cannula oxygen therapy versus conventional oxygen therapy and noninvasive ventilation on reintubation rate in adult patients after extubation: a systematic review and meta-analysis of randomized controlled trials. $J$ Intensive Care Med 2018;33.

44. Battisti A, Michotte JB, Tassaux D, et al. Non-invasive ventilation in the recovery room for postoperative respiratory failure: a feasibility study. Swiss Med Wkly 2005;135:339-43.

45. Chan AW, Tetzlaff JM, Altman DG, et al. SPIRIT 2013 statement: defining standard protocol items for clinical trials. Ann Intern Med 2013;158:200-7.
46. Hoffmann TC, Glasziou PP, Boutron I, et al. Better reporting of interventions: template for intervention description and replication (TIDieR) checklist and guide. BMJ 2014;348:g1687.

47. Boden I, Browning L, Skinner EH, et al. The LIPPSMAck POP (Lung Infection Prevention Post Surgery - Major Abdominal - with Pre-Operative Physiotherapy) trial: study protocol for a multi-centre randomised controlled trial. Trials 2015;16:573.

48. Boden I, Skinner EH, Browning L, et al. Preoperative physiotherapy for the prevention of respiratory complications after upper abdominal surgery: pragmatic, double blinded, multicentre randomised controlled trial. BMJ 2018;360:j5916.

49. Brooks D, Parsons J, Newton J, et al. Discharge criteria from perioperative physical therapy. Chest 2002;121:488-94.

50. Beasley R, Chien J, Douglas J, et al. Thoracic Society of Australia and New Zealand oxygen guidelines for acute oxygen use in adults: 'Swimming between the flags'. Respirology 2015;20:1182-91.

51. Agostini P, Naidu B, Cieslik H, et al. Comparison of recognition tools for postoperative pulmonary complications following thoracotomy. Physiotherapy 2011;97:278-83.

52. Sopena N, Sabrià M. Neunos 2000 Study Group. Multicenter study of hospital-acquired pneumonia in non-ICU patients. Chest 2005;127:213-9.

53. Rabin R, de Charro F. EQ-5D: a measure of health status from the EuroQol Group. Ann Med 2001;33:337-43.

54. Groll DL, To T, Bombardier C, et al. The development of a comorbidity index with physical function as the outcome. $J$ Clin Epidemiol 2005;58:595-602.

55. Rankin SL, Briffa TG, Morton AR, et al. A specific activity questionnaire to measure the functional capacity of cardiac patients. Am J Cardiol 1996;77:1220-3.

56. Vather R, Trivedi S, Bissett I. Defining postoperative ileus: results of a systematic review and global survey. J Gastrointest Surg 2013;17:962-72.

57. Glossop AJ, Shephard N, Shepherd N, et al. Non-invasive ventilation for weaning, avoiding reintubation after extubation and in the postoperative period: a meta-analysis. $\mathrm{Br} \mathrm{J}$ Anaesth 2012;109:305-14.

58. Roberts $C$, Torgerson DJ. Understanding controlled trials: baseline imbalance in randomised controlled trials. BMJ 1999;319:185.

59. Bickenbach KA, Karanicolas PJ, Ammori JB, et al. Up and down or side to side? A systematic review and meta-analysis examining the impact of incision on outcomes after abdominal surgery. Am J Surg 2013;206:400-9.

60. Severgnini P, Selmo G, Lanza C, et al. Protective mechanical ventilation during general anesthesia for open abdominal surgery improves postoperative pulmonary function. Anesthesiology 2013:118:1307-21.

61. Corcoran T, Rhodes JE, Clarke S, et al. Perioperative fluid management strategies in major surgery: a stratified meta-analysis. Anesth Analg 2012;114:640-51.

62. Nobili C, Marzano E, Oussoultzoglou E, et al. Multivariate analysis of risk factors for pulmonary complications after hepatic resection. Ann Surg 2012;255:540-50.

63. Peyton PJ, Myles PS, Silbert BS, et al. Perioperative epidural analgesia and outcome after major abdominal surgery in high-risk patients. Anesth Analg 2003;96:548.

64. Waller HM, Connor SJ. Cumulative sum (Cusum) analysis provides an objective measure of competency during training in endoscopic retrograde cholangio-pancreatography (ERCP). HPB 2009;11:565-9. 